\title{
Los Educadores Sociales en los centros de Educación Secundaria de Extremadura
}

\author{
David Galán Carretero \\ ies Tierrablanca. La Zarza (Badajoz)
}

\section{Resumen}

El educador social en los centros de enseñanza de Extremadura se entiende como una figura profesional que pretende dar respuesta a diversas necesidades de intervención socioeducativa, con el objeto de ofrecer una labor profesional adaptada a las necesidades de los alumnos y del propio sistema educativo, y aportar una visión complementaria a la educación académica. Esto no sólo garantiza la formación de alumnos, sino que además forma personas y ciudadanos dispuestos a enfrentarse a las realidades actuales.

Las funciones de los educadores sociales implican a toda la comunidad educativa con el fin de conseguir una educación integral para nuestros alumnos.

Palabras ClaVe: Educador Social, Intervención socioeducativa, Instituto de Enseñanza Secundaria, Absentismo escolar, Transporte escolar, Resolución de conflictos y mediación, Comunidad Educativa y Servicios Sociales.

\section{Summary}

The social educator in the education centres of Extremadura is considered as a professional figure who tries to give an answer to the various necessities of the socioeducational interacción, with the main aim of offering a professional task adapted to the necessities of the students and the educational system itself, and adopting a complementary view to the academic education. This not only guarantees the students' training, but also educates people and citizens willing to face the present realities. The social educators' functions involve all the educative community so as to attain a complete education for our students.

KeY WORDS: Social Educator, socio-educational intervention, secondary school, school absenteeism, school buses, conflict resolution and mediating, educational community and social services.

L a Educación Social en Extremadura no es una profesión nueva, ni tan sólo se viene desempeñando desde su implantación en la universidad de esta región. Hace ya más de dos décadas que existe la figura del educador en la Administración autonómica. Su andadura comenzó en los Centros de Atención a Menores, y contó desde ese momento con un reconocimiento formativo al exigir, para el ejercicio de la profesión, una titulación universitaria de grado medio.

Años más tarde, una vez consolidado el trabajo de los educadores en diversos centros, no sólo de atención a menores, sino 
también en centros de reforma y de educación especial, surge la necesidad de complementar la educación académica y formal con la actividad socioeducativa que desde la Educación Social se puede ofrecer.

Junto con la evidente necesidad de incorporar esta actividad a los centros, esta iniciativa se ve refrendada por el propio presidente de la Junta de Extremadura, quien en un acto público puso de manifiesto su intención y compromiso de añadir a las plantillas de los centros de Educación Secundaria, la figura de un Educador Social (en adelante E. S.) con el objeto de encaminar la educación formal de los alumnos hacia una educación integral.

Recién iniciado el curso académico 20022003, entre los meses de septiembre y octubre, los E. S. se fueron incorporando a los Centros de Secundaria. Como todo nuevo proceso de creación de figuras profesionales y funciones que integrar en un engranaje complejo, en este caso el de la educación formal, este no ha estado exento de preocupaciones y continuos intentos de reajuste.

\section{Integración orgánica y funcional de los Educadores Sociales en los Centros de Enseñanza Secundaria}

El proceso de contratación de ES para los Centros de Enseñanza Secundaria se inició en septiembre de 2002. Para el acceso a tales puestos de trabajo se exigió estar en posesión de la Diplomatura de Educación Social. Esto no sólo supuso un importante incremento cuantitativo en puestos de trabajo para quienes estaban en posesión de este título académico, por cuanto se contrataban alrededor de 140 ES, sino también cualitativo, pues Extremadura se ponía a la cabeza estatal de nuestra profesión creando un nuevo ámbito de trabajo, hasta el momento, inexplorado desde esta perspectiva.

El ES, en su relación contractual con la Junta de Extremadura, está asociado al per- sonal de administración y servicios, asemejándose en las condiciones laborales a administrativos, conserjes y personal de limpieza, cuya labor, aun siendo personal de los centros de enseñanza, está alejada de la intervención educativa directa y de la educación formal.

Así pues, los ES, al formar parte del personal de administración y servicios del centro, están integrados en la plantilla del Centro de Secundaria, pero dependen orgánicamente del secretario del centro, como profesor que ejerce la jefatura de este personal. Esta situación obliga a establecer relaciones entre este nuevo profesional y los demás profesionales con los que ha de trabajar de forma coordinada, con el objeto de complementar el proceso educativo de los alumnos.

Aun así, parece que se pretende dejar patente que la tarea profesional del ES debe estar enmarcada dentro del Departamento de Orientación, sumando sus conocimientos y habilidades a las intervenciones concretas que precisan los alumnos con necesidad específica de apoyo educativo y al resto de alumnos en general. De esta manera, el ES trabaja bajo la dependencia funcional e instrucción directa del Equipo Directivo, sobre todo en estrecha relación con la Jefatura de Estudios, coordinado convenientemente con los tutores y demás profesores, y compartiendo funciones con el Departamento de Orientación y el Departamento de Actividades Complementarias y Extraescolares.

\section{Justificación de la incorporación de los Educadores Sociales a los Centros de Enseñanza Secundaria de Extremadura}

La Consejería de Educación, Ciencia y Tecnología, en octubre del año 2002 y coincidiendo con la reciente adscripción de los ES a los centros, dicta unas Instrucciones ${ }^{1}$, a través de su Dirección General de 
Ordenación, Renovación y Centros, justificando la incorporación de estos profesionales, y estableciendo una primera aproximación a las funciones y ámbitos de actuación que pretenden sean abordados por esta nueva figura profesional.

Con la constante pretensión de avanzar hacia una educación integral de todos los alumnos, el hecho de integrar las tareas y actuaciones de un ES a los centros responde, en consecuencia, a la precisión de favorecer una respuesta educativa global, involucrando a todos los segmentos educativos, centrales y adyacentes, y sociales que se precisen para conseguirlo.

De un modo más concreto, esta nueva dotación profesional se argumenta basándose en la convicción de que los Institutos de Secundaria manifiestan una realidad socioeducativa cada vez más compleja, que plantea nuevos problemas que precisan de profesionales que los entiendan en profundidad y que, por su formación específica, puedan ayudar a prevenirlos y a buscar soluciones en colaboración con todos miembros de la Comunidad Educativa.

Estas dificultades se reflejan con mayor claridad en la Educación Secundaria Obligatoria, debido a las características psicológicas, sociales y familiares que presentan los adolescentes. Por otra parte, es especialmente inevitable la interconexión educativa con las familias, que, aún en estas edades, son actores principales en el desarrollo integral de sus hijos, por lo que es indispensable fomentar cauces de transmisión que perfeccionen la intervención conjunta entre el centro escolar y la familia, sobre todo en aquellos casos en los que las circunstancias familiares pudieran perjudicar de manera contraproducente el progreso de los educandos.

Otro de los aspectos que hay que destacar es la importancia de tener en cuenta los contextos en los que se encuentran inmersos los propios centros educativos. La co- munidad en la que se ubican cuenta con una red, más o menos amplia, de instituciones y recursos que, además, pueden ser aprovechados por toda la Comunidad Educativa.

Todos estos factores justifican la incorporación de los ES a los Centros de Enseñanza Secundaria, con el fin de afianzar la profesionalidad y la calidad de la enseñanza en la Comunidad Autónoma de Extremadura.

Aun así, "la necesidad de que el profesional de la educación social participe en el sistema educativo se justifica por sí sola con la pretensión de alcanzar, entre la propia formación escolar y la ineludible educación familiar, un proceso educativo integral adaptado a las necesidades individuales y capaz de enfrentarse a los retos sociales". (Galán, 2007, 28).

\section{Regulación legislativa de las funciones de los Educadores Sociales en los Centros de Enseñanza Secundaria}

Actualmente, las funciones de los ES vienen reguladas en dos documentos reglamentarios de carácter autonómico. De una parte, en el V Convenio Colectivo para el personal laboral al servicio de la Junta de Extremadura. Y de otra, la Instrucción de la Consejería de Educación por la que se concreta la Organización y Funcionamiento de los Centros de Enseñanza Secundaria.

El V Convenio Colectivo recoge, con carácter general, las condiciones laborales y funcionales por las que se rige el personal con contratación de esta naturaleza. La resolución de la Dirección General de Trabajo por la que se aprueba dicho convenio concreta las funciones de los ES en los centros educativos de la siguiente manera: "En los Institutos de educación secundaria (IES e IESO) se integra funcionalmente en el departamento de orientación ocupándose, en coordinación con éste y con los tutores, de la detección de 
factores de riesgo que puedan derivar en situaciones socioeducativas desfavorables, media en conflictos escolares, colabora en la prevención y control del absentismo escolar y en el cumplimiento de las normas de permanencia del alumnado de ESO en el centro durante la jornada escolar, participa junto al tutor en el desarrollo de habilidades sociales, se encarga de la recepción del alumnado de transporte escolar y de informar al equipo directivo de las incidencias ocurridas en dicho transporte escolar y diseña y vela por la ejecución de actuaciones encaminadas a favorecer la convivencia escolar, implicando a los distintos sectores de la comunidad educativa y a ésta con el entorno social en que está inmersa.

En los Centros a los que asistan alumnos procedentes de otras localidades, se ocupará también de atender a aquellos teniendo en cuenta, de cara a su integración, las incidencias que pudieran producirse con motivo de su llegada y salida del Centro".

Por su parte, y de un modo bastante más explícito y concreto, la propia Consejería de Educación dicta expresamente una Instrucción2 por la que se concreta la Organización y Funcionamiento de los Centros de Enseñanza Secundaria, y en la que se hace referencia a la figura del ES de este modo:

[...] el Educador Social se integrará funcionalmente en el Departamento de Orientación, participando desde sus propias responsabilidades profesionales en las funciones que a dicho Departamento se le asignan en el Reglamento Orgánico de los Institutos y colaborando asimismo en el desarrollo de las funciones del Departamento de Actividades Complementarias y Extraescolares.

Las funciones del Educador Social serán preferentemente la detección y prevención de factores de riesgo que puedan derivar en situaciones educativas desfavorables, la mediación en conflictos escolares, la colaboración en la prevención y control del absentismo escolar y en el cumplimiento de las normas de permanencia de los alumnos de ESO en el recinto escolar y la participación en el desarrollo de habilidades sociales. Asimismo, se consi- dera esencial su figura en el desarrollo del nuevo Plan Regional de Convivencia.

Además se encargará, bajo la coordinación del Director, del transporte escolar, supervisando la recepción y salida de los alumnos y comunicando al Equipo Directivo cualquier incidencia que pueda producirse colaborando en el buen funcionamiento de este servicio en los términos señalados en el Decreto 7/2004 de 10 de febrero (DOE del 17 de febrero) y en la Orden de 19 de diciembre de 2005 (DOE del 5 de enero de 2006).

Sin perjuicio de las funciones aludidas en el punto anterior, atenderán a la prevención y, en su caso, a la atención de situaciones consecuencia de la violencia de género en los términos que se contemplan en la Ley Orgánica 1/2004, de 28 de diciembre, de Medidas de Protección Integral contra la Violencia de Género.

A instancias del Director, el Educador Social podrá participar, con voz pero sin voto, en las sesiones de los órganos colegiados de gobierno de los centros y en las comisiones que se formen en el seno de éstos cuando se traten asuntos relacionados con sus funciones o así lo estime el Director. En todo caso, formará parte como miembro de pleno derecho de la comisión de convivencia del Consejo Escolar a la que se refiere el art. 8 del R.D. 732/1995 de 5 de mayo, por el que se establecen los derechos y deberes de los alumnos".

\section{Funciones de los Educadores Sociales en los Centros de Enseñanza Secundaria de la Comunidad Autónoma de Extremadura}

Teniendo en cuenta la descripción legislativa que se ha expuesto anteriormente, a continuación se detalla cómo se materializa el trabajo de los ES en la realidad de los centros.

\section{Contribución a los documentos de organización y funcionamiento de los centros}

Las tareas del ES, al igual que las de los docentes, deben quedar recogidas en las 
Programaciones Generales que se realizan a principio de curso. Teniendo en cuenta que el ES pertenece al Departamento de Orientación, su trabajo debe reflejarse, al comienzo del curso, en la programación de dicho departamento. Para ello, como ES que trabaja por la convivencia y la prevención, abarcando un amplio abanico de posibles conflictos, de las circunstancias específicas de la población adolescente, es preciso realizar un análisis de las necesidades que puedan darse en el centro.

En este caso, al igual que en otros campos de la Educación Social, es preceptivo que dicha programación se adapte a esta realidad, no sólo por ajustar y programar el trabajo basándose en unas carencias o en la prevención de las mismas, sino porque el mismo proyecto educativo de centro recoge los valores, los objetivos y prioridades de actuación $n^{3}$, teniendo en cuenta las características del entorno social y cultural, y realiza una valoración de las circunstancias socioeconómicas y familiares del entorno en que se encuentra y de la población a la que acoge.

Además, puesto que su labor está relacionada directamente con la convivencia, el ES debe elaborar propuestas de programas y actuaciones encaminadas a mejorarla y prevenir los conflictos, así como redactar planes específicos para el fomento de las relaciones de los diferentes sectores de la comunidad educativa, y establecer reciprocidades entre el centro y el entorno en el que se encuentra inmerso a nivel institucional, político y social.

Igualmente, se tiene en cuenta la manera en la que se van a abordar cada una de las cuestiones de convivencia, quiénes son las personas y sectores implicados, los objetivos que se pretenden conseguir, así como el modo de ejercer la coordinación y de qué forma se van a evaluar los resultados obtenidos.

Todas estas propuestas de organización y trabajo, adaptadas a las particularidades de cada centro, son incorporadas tanto al Proyecto Educativo como a la Programación General Anual, basándose en la singularidad que permite la Ley de Educación con respecto a la autonomía pedagógica de los centros.

\section{Coordinación profesional en el centro educativo}

El trabajo en la enseñanza supone una labor de equipo, y la educación integral de los alumnos es un trabajo conjunto en el que debe estar implicado todo profesional que trabaje en el centro, tanto el personal docente como el personal no docente, que no por ello deja de ser educativo.

Las relaciones de coordinación del ES se establecen de diferentes maneras. El hecho de pertenecer funcionalmente al Departamento de Orientación ya supone la inevitable coordinación con el resto de los profesionales que allí se encuentran. Además, las propias funciones que se atribuyen a los ES en relación con este departamento, le hacen partícipe del desarrollo del mismo, tales como la asistencia a sus reuniones y el desarrollo conjunto de intervenciones propias para detectar y prevenir los riesgos que puedan derivar en situaciones sociales y educativas desfavorables.

Del mismo modo, aunque sin pertenecer orgánicamente al Departamento de Actividades Complementarias y Extraescolares, participa en el desarrollo de las funciones propias de este departamento, aportando su labor técnica, no sólo en la elaboración de propuestas, sino también en el conveniente desarrollo de las mismas. El modo de hacer efectiva esta coordinación es mediante las reuniones semanales que tienen estipulados estos departamentos, y a través de otros momentos que se puedan establecer para conjugar y complementar las distintas intervenciones profesionales.

Con respecto al Equipo Directivo, la relación de coordinación es especialmente 
relevante por dos motivos. En primer lugar, porque el ES debe tener en cuenta todos los aspectos que se recogen en el Proyecto Educativo de cada centro al plantear sus intervenciones, priorizando y atendiendo aquellos objetivos que la dirección del centro pretende conseguir y considera especialmente importantes para el desarrollo integral de los alumnos, y para lograr una atención global ante sus necesidades educativas. En segundo lugar, hay que tener en cuenta que es crucial la coordinación bidireccional entre la Jefatura de Estudios y el ES, ya que es una de las principales vías de constante información y detección de alumnos absentistas y conductas contrarias a las normas de convivencia del centro, y donde el profesional de la educación social puede y debe aportar su visión reeducadora al procedimiento de corrección y resolución positiva de dichos comportamientos.

La relación con los tutores también encuentra su propio espacio. Los profesores que ejercen la tutoría de los grupos cuentan con el apoyo del ES, no sólo para colaborar en las actuaciones encaminadas a mejorar la convivencia y evitar el absentismo, sino además para favorecer el desarrollo de tutorías específicas en materias especializadas. Para ello, el tutor cuenta con dos periodos lectivos complementarios que puede utilizar para contar con la colaboración, entre otros, del ES

Asimismo, el profesional de la Educación Social se ofrece como recurso al resto de los profesionales del instituto y está dispuesto a establecer espacios de coordinación de cara a aportar modelos de intervención con los alumnos, e incluso planteamientos de trabajo complementarios, que ayuden a mejorar la calidad de la enseñanza en los diferentes periodos lectivos.

Por último, es preciso destacar que la actividad del ES también se incluye en los órganos colegiados de gobierno, claustro de profesores y consejo escolar, y en las comisiones que de estos puedan derivarse, cuando el director lo estime oportuno, por la relación que pudieran tener los temas tratados con la labor de este profesional. En este caso, el ES participa en dichos órganos con voz pero sin voto, a excepción de la Comisión de Convivencia, en la que se integra como miembro de pleno derecho.

\section{Control del absentismo escolar}

Una de las labores de mayor importancia del ES es su actuación sobre el absentismo escolar de los alumnos escolarizados en los cursos de Secundaria Obligatoria. Para ello, este profesional se acoge al protocolo establecido para estos casos en la legislación autonómica ${ }^{4}$, mediante la que se le atribuyen tareas de intervención y control ante las conductas absentistas, en coordinación con todos los implicados en la prevención del absentismo: tutores, Equipo Directivo, Técnico de Servicios a la Comunidad, Trabajador Social y Educador Social de los servicios sociales de base, etc., con el objeto de unificar los métodos de intervención.

En los casos en los que el normal protocolo de control de las faltas no es suficiente para solventar la situación, la Jefatura de Estudios cuenta con la intervención del ES para que, en una entrevista con la familia del menor y el tutor del curso, se pueda informar a estos de la situación y recabar la necesaria colaboración de los padres o representantes legales. El propósito final de dicha entrevista es comunicarles las consecuencias que pueden derivarse en el caso de persistir esta situación, y poner en su conocimiento la responsabilidad que tienen como padres de cooperar y ser partícipes en la búsqueda de soluciones.

En los centros educativos se crean Comisiones de Absentismo de las que el ES forma parte, contribuyendo al control y seguimiento en esta materia, y elaborando 
e interviniendo en los respectivos Programas Individuales de Intervención, junto con los tutores y profesores de compensación educativa, ajustados a las peculiares características de cada caso.

Asimismo, también se encomienda que el ES sea el profesional encargado de establecer la correspondiente coordinación de las actuaciones con los Servicios Sociales en aquellos casos en los que pueda detectarse alguna situación de desprotección.

\section{Relaciones con las familias}

La necesaria relación con las familias de los alumnos viene dada no sólo por las implicaciones que las actuaciones del ES tengan, sino porque, y muy fundamentalmente, los padres no pueden declinar nunca su responsabilidad como tales cuando desde los centros de enseñanza se les transmite información sobre el desarrollo educativo de sus hijos.

Salvando estas cuestiones, la labor del ES en su trabajo directo con los padres podría centrarse en tres aspectos concretos. En primer lugar, como parte implicada en la intervención de los alumnos. No en todos los casos es preciso, ni todas las situaciones lo aconsejan, pero un porcentaje alto necesita que la familia se involucre en el proceso y participe en la intervención individualizada; de este modo se pretende tener mayores garantías de éxito al poder intervenir también con los padres, haciendo extensivo el proceso educativo más allá de los límites académicos.

En segundo lugar, existe una labor importante que el ES comparte con lo académico y es el fomento de la participación de las familias en la vida del centro educativo, sus órganos de coordinación y gobierno, y su implicación en los instrumentos de representación de madres y padres. Las AMPAS ${ }^{5}$, como instrumento por antonomasia de representación de padres en el ámbito escolar, son uno de los medios, generalmente existente en todos los centros, desde los que poder atraerlos, bien para formar e informar en cuestiones académicas, bien como simple estructura donde compartir experiencias sobre el día a día con sus hijos.

Desde esta posición, y teniendo en cuenta que los alumnos son el eje que nos une a las familias, no conviene olvidar que las asociaciones de alumnos también son un medio al que el ES puede hacer múltiples aportaciones: formativas e informativas. Así, puede ponerles en contacto con otras instituciones o recursos de ocio y tiempo libre, tales como el propio Departamento de Actividades Complementarias y Extraescolares, o los centros de ocio de la comunidad en la que se ubican.

Por último, en tercer lugar, y no por ello menos importante, cabe destacar la especial relevancia de las Escuelas de Padres como lugares de formación e intercambio de experiencias. El ES puede, y debe, contribuir al desarrollo de las mismas, apoyando a las AMPA a organizar estas sesiones, incentivando la participación, colaborando en la búsqueda de profesionales y expertos en materias de interés para los padres, e incluso impartiendo conferencias y debates como profesionales de la actividad socioeducativa en aspectos específicos de prevención e intervención personal y social.

Por tanto, el ES no debe perder uno de los motores activos de los centros, las familias, aun siendo conscientes de las dificultades que ello entraña, sobre todo para aquellas que más precisan de estos recursos.

\section{El Educador Social y las Instituciones de la comunidad}

El ES, por el hecho de trabajar en un centro educativo, no tiene que aislarse, ni limitar su intervención al recinto escolar, ya que precisamente su labor socioeducativa, a diferencia de la educación formal, más 
pegada a lo académico, va mucho más allá, pudiendo coordinarse y hacer uso de los recursos externos y relacionados con el instituto.

No obstante, mantener las relaciones del instituto con su entorno inmediato no es una función exclusiva del ES, ya que es competencia primera del director del centro. El ES debe tener en cuenta que, aunque pretenda dar la mayor cobertura posible a las situaciones socioeducativas que vienen derivadas de su figura profesional, no puede abarcar todos los ámbitos posibles, ni desde la posición que ocupa en su centro de trabajo, ni por las responsabilidades que tiene atribuidas.

En este sentido, es básico establecer relaciones con los Servicios Sociales de la localidad y/o localidades de procedencia de los alumnos, ya que a través de estos se pueden establecer procesos de intervención más amplios y complementarios, con el objeto de intentar prevenir en su sentido más extenso y conseguir una actuación integral sobre todos los aspectos que rodean a los menores y sus familias.

Para ello resulta primordial que el ES establezca cuantos mecanismos sean precisos para crear y dar fluidez a las vías de información e intercambio, que agilicen todos los procesos, concretando espacios de interacción y tiempos periódicos para reuniones conjuntas. En la mayoría de los casos, una intervención coordinada logra mejor pronóstico de implicación de las partes afectadas o en riesgo, además de ofrecer un mayor abanico de posibilidades de acción socioeducativa.

Asimismo, el Servicio de Protección del Menor constituye un recurso que el ES debe conocer a fondo, consciente de la importancia del mismo en la detección y notificación de casos en circunstancias de riesgo y/o desprotección. Este servicio puede ser utilizado por el ES mediante contacto directo, no sólo como profesional, sino también como persona sensible a las dificultades sociales, o a través de la mediación de los Servicios Sociales municipales, encargados de canalizar estas demandas.

Por otra parte, los Servicios Sanitarios no sólo contribuyen a mantener actualizado el calendario de vacunación, sino que es necesaria una escrupulosa reciprocidad entre el ES y el Servicio de Salud, de cara a detectar individual y/o conjuntamente determinados factores de riesgo y desprotección, así como cualquier enfermedad que pueda afectar al normal desarrollo de los menores.

En este caso, al igual que con los Servicios Sociales, es preciso establecer unos cauces de comunicación y espacios de reunión para trabajar al unísono hacia unos fines comunes.

Con el objeto de poder unificar criterios, hay que tener en cuenta que es importante establecer unos protocolos de actuación con estas instituciones, con el objeto de evitar duplicidad de intervenciones o solapar actuaciones que intercepten la premura de acción que en ocasiones se demanda.

Lo que está claro es que el ES, en relación con estos recursos, es una pieza clave de coordinación e información. Puesto que es preceptivo para todos los menores matricularse en la Educación Secundaria Obligatoria, entra dentro de lo posible que, en algún momento determinado, en el quehacer diario de los centros educativos, tanto este profesional como cualquier otro de los que allí trabajan puedan detectar situaciones que precisen de intervención específica.

Otro aspecto que el ES debe trabajar con fluidez en estos casos son los informes socioeducativos. Estos son un elemento indispensable por medio del cual se puede dejar constancia del trabajo realizado en todo momento, y del traspaso de información, en todo caso confidencial, de cada uno de los casos con los que se intervenga, 
evitando perder efectividad en los canales de intercambio.

También hay que tener en cuenta que, además de los recursos que puedan ofrecer las administraciones, existe una amplia oferta de profesionales en el marco del ámbito privado que complementa y subsana las carencias de prestaciones que el propio sistema educativo, en particular, y público, en general, no puede asumir.

Psicólogos, logopedas, médicos especialistas, o gabinetes psicopedagógicos, entre otros, son algunos de los recursos especializados que, en caso de ser utilizados por nuestros alumnos en aspectos relacionados con las tareas propias del ES, exigen una necesaria coordinación que complemente ambas intervenciones profesionales.

Por otro lado, no todas las relaciones institucionales están vinculadas con la intervención socioeducativa, por lo que también hay que tener en cuenta aquellas que directa o indirectamente se relacionan con estas y que pueden ofrecer alternativas de ocio y tiempo libre. En este caso, los propios Ayuntamientos cuentan con educadores sociales, animadores socioculturales, o monitores de actividades extraescolares, entre otros, que en la medida de lo posible plantean la dinamización de menores y jóvenes, creando espacios para la ocupación del ocio y el tiempo libre, aportando unos valores afectivos y socioeducativos con el fin de inculcar nuevos modelos de convivencia.

De esta manera, el ES del centro educativo cuenta con estos recursos para poder utilizarlos desde dos vertientes. Una, como simple oferta de ocio para los alumnos. Otra, como prolongación de su propia intervención socioeducativa en el centro.

Se tendrán en consideración estas actividades de cara a poder ser utilizadas para integrar a los menores en la localidad, ofrecerles la posibilidad de ampliar su círculo de amigos, mejorar sus competencias sociales y personales, o simplemente desarrollar nue- vas aficiones. Son recomendables porque ofrecen la posibilidad de llevar la propia intervención más allá del centro educativo, pero con la salvedad de que en este caso se cuenta con otros profesionales que pueden supervisar y complementar de manera educativa las acciones que se llevan a cabo.

También aquí se precisa establecer una coordinación con estos servicios, para permanecer informados de la oferta de actividades que ofrece cada una de las instituciones. Igualmente, esta relación servirá para establecer y llevar a cabo intervenciones conjuntas y coordinadas con aquellos chavales con los que se decida, aportando una visión distinta de la que se trabaja desde el propio centro educativo.

Por último, hay que destacar que el instituto, como aglutinador de parte de la población a la que se dirige esta ocupación del tiempo de ocio, puede utilizar su posición privilegiada para sondear los intereses de los alumnos que acoge, de tal manera que se puedan plantear actividades ajustadas a sus demandas y garantizar ciertas posibilidades de éxito. Así, el ES del centro actúa como mediador entre los interesados y las instituciones que lo pueden poner en marcha.

\section{Participación de las responsabilidades y funciones del Departamento de Orientación y colaboración en las funciones del Departamento de Actividades Complementarias y Extraescolares}

Compartir las propias responsabilidades del Departamento de Orientación es otra de las funciones del ES, así como contribuir a planificar y desarrollar las del Departamento de Actividades Complementarias y Extraescolares.

Respecto al primero, el ES aporta su actividad profesional en aquellos aspectos de su competencia relacionados con el desarrollo personal, social y educativo de los alumnos, proporcionando elementos que favorezcan 
su educación, sus relaciones sociales, así como los aspectos familiares que pudieran influir positivamente y neutralizar aquellos que pudieran restar oportunidades al proceso educativo de los chavales.

En este sentido, y teniendo en cuenta esto, el ES, integrado en las funciones del Departamento de Orientación y junto a los miembros del mismo, realiza propuestas de organización y orientación educativa, colabora en la prevención y detección temprana de problemas de aprendizaje, contribuye a la planificación y desarrollo del Plan de Acción Tutorial y Plan de Orientación Académica y Profesional, realiza las evaluaciones de los alumnos en los aspectos concretos de su competencia, y coopera en la organización y realización de actividades complementarias. Además, en los institutos con residencia para estudiantes de secundaria, colabora con los profesionales responsables de la atención educativa de los alumnos internos.

Asimismo, con el Departamento de Actividades Complementarias y Extraescolares colabora igualmente en la planificación de actividades y viajes de estudios de carácter cultural, deportivo y artístico, realizadas en coordinación con los diversos sectores de la comunidad educativa o en aplicación de acuerdos con otras entidades. También contribuye a la organización y funcionamiento de la biblioteca en aquellas actividades relacionadas con la animación a la lectura. Y respecto a las residencias de estudiantes de secundaria, colabora en la organización del ocio y tiempo libre.

\section{Programas socioeducativos que desempeña el Educador Social}

Son múltiples los programas de intervención que puede llevar a cabo el ES, tanto de prevención como de intervención personal, familiar y socioeducativa. El trabajo de este profesional debe adaptarse continuamente a los cambios y necesidades que surgen en la dinámica propia de los centros educativos, ofreciendo las respuestas más adecuadas en cada momento y en función de los recursos con los que cuente. Por este motivo, no se pretende recoger aquí todos los posibles programas que pudieran desempeñarse, pero sí los más habituales en la generalidad de los centros educativos de la Comunidad Autónoma de Extremadura.

\subsection{Educación para la salud}

Además de contribuir al desarrollo de los programas de salud escolar con respecto a las vacunaciones propias de la edad y los exámenes de salud, el ES plantea actividades de prevención, tanto primaria como secundaria. En este sentido, la propia Consejería de Educación ofrece a los centros educativos recursos conjuntos con la Consejería de Sanidad y Consumo, aportando materiales para la realización de Campañas de prevención del tabaquismo en la escuela, o de prevención de la drogadicción como Prevenir para vivir, en coordinación con la Fundación de Ayuda contra la Drogadicción, y facilitando la asistencia a representaciones teatrales en torno a la prevención del sida, tal como La farsa del amor, entre otras.

Igualmente puede llevar a cabo otros programas de prevención de la salud relacionados con la educación sexual, la alimentación, etc., dentro de lo que su propia formación personal le permita, ampliando el marco de actuación en esta materia contando con profesionales específicos de la salud, especialistas en temas que resulten de interés no sólo para los alumnos, sino también para los padres desde el espacio que ofrecen las escuelas de padres.

\subsection{Ocio y tiempo libre}

El ES, en contacto con las instituciones que implican a la comunidad educativa, explora 
los recursos de ocio y tiempo libre existentes de tal modo que pueda ofrecer alternativas viables y saludables a los alumnos del centro. Con ello también se pretende fomentar las relaciones que se crean en el instituto, así como dar continuidad a las intervenciones que allí se realizan, y que aconsejan el contacto con nuevos recursos socioeducativos paralelos al centro educativo.

\subsection{Educación para la convivencia}

Se realizan múltiples programas en este sentido. Algunos se trabajan en pequeños grupos, otros abarcan a los miembros de una clase y otros pretenden implicar a todo el instituto.

Desde este tipo de programas se trabaja por la convivencia pacífica y educativa en los centros, educando en la tolerancia, contra el racismo y la xenofobia, fomentando las relaciones de respeto mutuo entre todos los miembros de la comunidad educativa.

Para esto, en el centro se trabajan las normas de convivencia que regulan la vida académica y el sisterna sancionador al que el ES pretende imprimir un carácter reeducador, todo ello apoyado por actividades trarısversales en cada materia curricular.

También se propone, en conjunción con el Departamento de Actividades Complementarias y Extraescolares, días del centro para fomentar las relaciones de cuantas personas conviven en el mismo, y días étnicos y culturales, desde los que se pueder. articular actividades relacionadas con las distintas etnias y costumbres.

Otro de los puntos fuertes que el ES apoya en relación a la convivencia es la prevención de la viclencia de género. Para ello, la propia Consejería de Educación propone realizar dos actividades por grupo de clase al trimestre con la intención de combatir las actitudes que fomenten la violencia de género; en estas actividades, el ES tiene un papel especialmente relevante.
Desde esta perspectiva, el ES realiza las aportaciones que sean precisas tanto al Proyecto Educativo como a la Programación General Anual, de tal manera que la convivencia constituya uno de los ejes centrales desde el que poder ejercer la educación de los alumnos.

Y hablando de convivencia, no se puede olvidar la integración de los alumnos que llegan nuevos al centro, bien porque vienen del colegio, bien procedentes de otras localidades. Es fundamental que a lo largo de los primeros días se les explique cuál es el funcionamiento del centro, horarios, normas de convivencia, etc., haciéndoles partícipes desde el primer momento de la vida escolar, y provocando actividades que permitan que estos nuevos alumnos puedan ir imprimiendo su propio carácter a los espacios comunes.

\subsection{Absentismo escolar}

El absentismo es uno de los principales problemas a los que se enfrenta la educación obligatoria. En este sentido, y paralelo al protcicolo descrito anteriormente con el fin de erradicar estos casos, e. ES realiza intervenciones con los alumnos y las familias. Para ello, el trabajo con los propios menores se centra en averiguar las zausas que están provocando la inasistencia al centro escolar, para después buscar soluciones conjuntas y adecuadas a las expectativas de los propios alumnos. En muchas ocasiones, esto conlleva una labor más allá de la intervención irıdividual, involucrando a profesores, pad:es e incluso compañeros de clase para incentivar al menor y que desista de su actitud.

Con las familias, el trabajo no es menos irnportante. En muchos casos las habilidades parentales pueden lleçar a ser deficitarias, poco motivadoras e incluso coercitivas, resultando inadecuadas de cara a encontrar una solución. Aportar habil dades y pautas de relación y atención a los padres es básico 
cuando se pretende que la intervención pueda complementarse en el ámbito familiar.

En aquellos casos en los que incluso las propias familias no muestran interés alguno por colaborar, el ES toma contacto con los recursos pertinentes, siguiendo el protocolo establecido para ello, con objeto de ampliar las posibilidades de resolución al contar con los Servicios Sociales municipales de la localidad, y autonómicos si fuera preciso.

\subsection{Modificación de conducta}

Estos programas de conductas están dirigidos principalmente a alumnos que presentan conductas disruptivas en el aula y a aquellos con problemas graves de conducta dentro y fuera de la clase. En estas situaciones es primordial el trabajo coordinado entre el ES y el equipo educativo del grupo-clase, de manera que los profesores, como afectados ante la imposibilidad de poder atender sus clases en unas condiciones de calidad para todos los alumnos, se involucren en actividades tales como el control del comportamiento y la administración de refuerzos o extinción de los mismos, ante las diferentes manifestaciones conductuales de estos alumnos.

Por otra parte, el papel de las familias es igualmente fundamental, pues, sin el apoyo de las mismas, es muy complicado obtener resultados positivos, ya que son precisamente ellos los encargados de dar continuidad al trabajo que se realiza en el instituto y de gestionar las consecuencias derivadas de tales actitudes en el ámbito extraescolar.

La relación que el ES establece con los menores es diferente a la que pueden mantener con sus profesores, ya que no trata de evaluar ni sancionar su futuro académico, sino que pretende explotar sus cualidades y ampliar las expectativas sobre sus propias metas. Es una relación más equitativa con el objeto de acercar posiciones de cara a un mayor entendimiento, salvando las distancias pertinentes entre ES y educando.

\subsection{Desarrollo de habilidades}

Los trabajos concretos para mejorar las competencias de los alumnos se articulan de diferentes maneras en función de la casuística. En general, el ES realiza estas acciones sobre las habilidades sociales de los alumnos, dedicando los tiempos apropiados con el objeto de conseguir unas mejores relaciones sociales de los chavales, fomentar un clima de entendimiento, tanto en el ámbito escolar como familiar, y optimizar aquellos aspectos que el autoconcepto limite, para conseguir unas relaciones y un sentimiento sobre sí mismo en perfecta armonía.

Los intentos de generalización de los aprendizajes que forman parte de las sesiones de habilidades sociales implican nuevamente a toda la comunidad educativa (padres, profesores y resto del personal del centro), lo que requiere también en estos casos la precisa coordinación que contribuya al éxito de la intervención.

Es necesario hacer mención, en este caso, de la intervención que el ES desempeña hacia los alumnos con necesidad específica de apoyo educativo, con el propósito de conseguir sacar el máximo partido a sus potencialidades personales facilitando su integración en el mundo familiar, social y laboral.

Su labor no se centra en aspectos curriculares, sino que trata de reforzar las habilidades que puedan estar dificultando su desarrollo personal y su integración social como cualquier otro alumno, preferentemente de aquellos que presentan necesidades educativas especiales, los que se están integrando de forma tardía al sistema educativo español, o aquellos que necesitan recursos de compensación de desigualdades como resultado de componentes sociales, económicos, étnicos, etc.

Se debe destacar también el apoyo que ofrece a los padres aportando habilidades y estrategias que pueden utilizar para mejo- 
rar sus relaciones paternofiliales, ya que a veces demandan atención especializada al verse desbordados por los comportamientos de sus propios hijos. Estas intervenciones pueden realizarse directamente con el ES o bien integradas dentro del marco de la Escuela de Padres.

\subsection{Fomento de la igualdad}

El ES integrado en un centro educativo continúa siendo un técnico de lo social, sensible a las situaciones desfavorables. Su labor de detección de casos con dificultades económicas, culturales o étnicas le sitúa en un lugar privilegiado para poder ofrecer los diferentes recursos de que dispone la comunidad.

No sólo se habla en estas situaciones de la igualdad de derechos, puesto que ya se da por hecho, sino de la pretensión de igualar los medios precisos para alcanzar la igualdad de acceso a la educación, en las mismas condiciones materiales que los demás alumnos.

Para ello, este profesional pone a disposición de la comunidad educativa toda la información necesaria para garantizar el acceso a becas y ayudas que ofrecen las distintas administraciones educativas. También está en disposición de ofrecer el contacto con los Servicios Sociales y otras entidades, a fin de facilitar la normalización de las circunstancias personales, familiares y sociales.

\subsection{Resolución de conflictos y mediación}

La resolución de conflictos en el ámbito escolar se viene desempeñando en muchos centros de la comunidad autónoma, en proyectos coordinados por ES, que arrojan, además, unos resultados muy positivos. En esta línea, se viene trabajando con los alumnos todo tipo de habilidades de resolución de conflictos, bien en pequeños grupos, bien a través de las tutorías e integrados en el Plan de Acción Tutorial. Algunas de estas activi- dades se incluyen dentro de amplios programas de habilidades sociales.

En marzo de 2007, la Consejería de Educación ha publicado un Decreto ${ }^{6}$ por el que se establecen los derechos y deberes de los alumnos y las normas de convivencia de los centros educativos. En él se refleja la necesidad de trabajar la resolución de conflictos, así como de crear la figura del mediador para la resolución conciliada de los mismos.

Concretamente, en este decreto se determina que las funciones de los ES respecto a esta materia serán de detección y prevención de factores de riesgo que pudieran derivar en situaciones desfavorables y de mediación en los conflictos garantizando imparcialidad, tratamiento educativo y confidencialidad.

Las funciones específicas que se le atribuyen al ES como mediador en los conflictos se refieren a intervenir en el proceso de mediación ayudando a comprender a las partes los intereses y aspiraciones para llegar a un buen entendimiento. Además, aconseja auxiliarse en la experiencia de funciones mediadoras previas, realizar el seguimiento de los casos y utilizar estrategias pedagógicas para el cumplimiento de lo acordado en el proceso de mediación.

\subsection{Apoyo al profesorado}

Finalmente se hace necesario destacar que el ES, entre sus funciones, acoge la capacidad de poder aportar al profesorado estrategias de trabajo y control del comportamiento, salvando las limitaciones curriculares, que contribuyan a favorecer la calidad educativa de las clases y el clima de las mismas.

Igualmente puede ofrecer modelos de trabajo concretos con algunos alumnos, en función de las características personales obtenidas mediante evaluación previa, así como aportar elementos de motivación que sirvan para mantener activa la voluntad de trabajo del alumno. 


\section{Coordinación del servicio de transporte escolar}

Los centros que reciben diariamente alumnos de otras localidades tienen una función más que añadir al profesional de la Educación Social: coordinar y supervisar el servicio de transporte escolar. En este sentido, se le encomienda la recepción diaria del alumnado transportado procedente de otras localidades, así como su acompañamiento hasta el momento de su subida al servicio de transporte al finalizar la jornada escolar.

A esta función, menos relacionada con la intervención directa y la labor profesional de un ES, se ha pretendido aportarle un elemento educativo, añadiendo a esta tarea, además de informar al centro y a las familias de las incidencias que pudieran producirse, la de programar y ejecutar, junto con el Equipo Directivo, tutores y Departamento de Orientación, las actuaciones precisas encaminadas a garantizar la información a las familias sobre el proceso académico de sus hijos, la vida en el centro y las actividades que en el mismo se desarrollan. Junto a esto, la tarea de supervisar este servicio conlleva una serie de trabajo de carácter administrativo y burocrático, que en ocasiones resta un tiempo que sería de gran valor si se dedicase a la atención de los alumnos.

Y hasta aquí la descripción de las tareas que conforman el día a día del ES en un Centro de Enseñanza Secundaria. Pero, antes de acabar, habría que destacar determinados aspectos generales que se han venido constatando desde la incorporación de esta figura profesional a los centros educativos.

Este conglomerado de tareas, funciones y responsabilidades de los ES se vienen estructurando desde septiembre de 2002. Hay que tener en cuenta el esfuerzo que la Administración autonómica ha realizado para diseñar un catálogo de funciones y tareas que desempeñar, salvando discrepancias y asignando funciones que en ocasiones no han agradado al colectivo de ES. Sin embargo, hay que destacar, por encima de todo, que son los propios ES los que han venido conformando sus labores diarias, adaptándolas a su centro particular y compartiendo entre los compañeros múltiples experiencias, que incluso hoy sirven de referencia a la propia Administración.

Las dificultades no han sido pocas, ya que no sólo había que ponerse a trabajar, sino que también hubo que darse a conocer e integrarse en la vida de los centros cuya dinámica, de carácter académico, no es fácil de reestructurar. La preocupación con la que los Equipos Directivos y Claustros veían su incorporación a los institutos se ha convertido en una relación de dependencia bidireccional, convirtiéndose el ES en un profesional con entidad propia, consiguiendo encajar todas sus responsabilidades y tareas por encima de relaciones contractuales con la Administración y diferencias profesionales con otros colectivos.

Finalmente, cabe decir que Extremadura también apuesta por una educación de calidad, pero quiere ir más allá. La política educativa de la comunidad autónoma no sólo pretende formar alumnos sino, que también quiere educar personas, aportando una formación que, además de facilitar su acceso al mundo laboral, cree nuevas conciencias, valores y ciudadanos responsables adaptados a la realidad de nuestro tiempo. Es una lucha por la tan ansiada educación integral, y en ello los ES parece que tienen mucho que ver. 


\section{Referencias bibliográficas}

Caride, J. A. (2002): "Educación Social como proyecto ético y tarea cívica", en Revista Interuniversitaria de Pedagogía Social, 9 (2002), pp. 91-126.

Castro Rodríguez, M.; Malhiero Gutiérrez, X. M., Rodríguez Rodríguez, J. (2006): A escola, ¿ipunto de encontro entre profesorado e educadores/as sociais? Santiago de Compostela. Nova Escola Galega. Colexio de Educadores Sociais de Galicia.

Galán Carretero, D. (2007): "Modalidades de participación del educador social en la escuela", en Aula de Innovación Educativa, 160 (2007), pp. 27-28.

\section{Notas}

${ }^{1}$ Instrucciones de la Dirección General de Ordenación, Renovación y Centros por las que se establecen las funciones y ámbitos de actuación de los Educadores Sociales en centros de Educación Secundaria de la red pública dependiente de la Consejería de Educación, Ciencia y Tecnología de la Junta de Extremadura.

${ }^{2}$ Instrucciones de la Dirección General de Política Educativa de 27 de Junio de 2006, por la que se concretan las normas de carácter general a las que deben adecuar su organización y funcionamiento los Institutos de Educación Secundaria y los Institutos de Educación Secundaria Obligatoria de Extremadura.

${ }^{3}$ Ley Orgánica 2/2006, de 3 de mayo, de Educación. Art. 121.

${ }^{4}$ Decreto $142 / 2005$, de 7 de junio, por el que se regula la prevención, control y seguimiento del absentismo escolar en la Comunidad Autónoma de Extremadura; Orden de 19 de diciembre de 2005 por la que se regulan las actuaciones de la consejería de educación para la prevención, control y seguimiento del absentismo escolar.

${ }^{5}$ Asociaciones de Madres y Padres.

${ }^{6}$ Decreto 50/2007, de 20 de marzo, por el que se establecen los derechos y deberes del alumnado y normas de convivencia en los centros docentes sostenidos con fondos públicos de la Comunidad Autónoma de Extremadura.

DiRECCIÓN DEL AUTOR: David Galán Carretero. Junta de Extremadura. IES "Tierrablanca". La Zarza (Badajoz).Correo electrónico: dglancar@yahoo.es

Fecha de recepción del artículo: 15.XI.2007

Fecha de aceptación definitiva: 10.XII.2007 



\section{INVESTIGACIÓN}

Valores y actitudes de los/as jóvenes andaluces en contextos pluriculturales

- ROCÍO CÁRDENAS RODRÍGUEZ .

Tipología de la dependencia de las personas con discapacidad atendidas en centros residenciales, de día y ocupacionales

- SAMUEL FERNÁNDEZ FERNÁNDEZ

Inserción laboral de jóvenes con discapacidad. Análisis de las prácticas laborales

- MARTINA PÉREZ SERRANO 
